\title{
The Proceed of Thinking Surrounded by Some French and Tunisian Gymnastic Teachers
}

\author{
Naila Bali ${ }^{1,2}$, Souhail hermassi ${ }^{1,2}$, Zied Hassen ${ }^{3,}$ Monoem Haddad ${ }^{2,4}$, Karim \\ Chamari $^{2,5}$ \\ ${ }^{1}$ (High Institute of Sport and Physical Education, Ksar Saîd, University la Manouba, Tunis, Tunisia). \\ ${ }_{2}^{2}$ (Tunisian Research Laboratory «Sport Performance Optimization », Tunis, Tunisia). \\ ${ }^{3}$ (National Observatory of Sport ONS Tunis, Tunisia). \\ ${ }^{4}$ (High Institute of Sport and Physical Education, Ke)f. \\ ${ }_{5}^{5}$ (Research and Education Centre, Aspetar, Qatar Orthopaedic and Sports Medicine Hospital, Doha, Qatar)
}

\begin{abstract}
Background: In reference to the anthropological theory of teaching of Chevallard, teacher's knowledge proved by his actions and his words. Our research questions "implicit thoughts" as determinants of teaching practices of gymnastic teachers. Aims: This study includes a qualitative analysis which aims to identify first the teaching practices of Tunisian and French gymnastic university teachers (TFGUT) and check in a second time the relationship between teaching practices teachers and their gymnastic "implicit thoughts. "Method: It's a descriptive lexploratory methodology based on observation and video recorded with twelve 12 sessions lasting one hour each and twelve semi structured interviews (40 minutes each) and gave teachers the opportunity to share their perspectives on broad topics such as education, teaching, and society, and also on more succinct topics such as individual students and situations that had occurred in previous lessons. Data collection: Data were collected during 7 months of observations and interviews with twelve TFGUT including 6 Tunisians and 6 French. They all teach not mixed class in Levell (first year, BAC+1). Result: TFGUT justify their teaching practices by the fact that the level of the students did not allow them to integrate science into the practice gym sessions. Conclusion: We ask later if our culture still steeped in the dichotomy "between theory and practice" can promote joint that positivism has promoted in the West in the late nineteenth century.
\end{abstract}

\section{Introduction}

The analysis of teaching practices by professionals has been a growing interest both in the training area in the work (Marks, 1990; Shulman \& Grossman, 1987) and Michigan State Universities (Ball \& McDiarmid, 1990; Fiman-Nemser \& Parker, 1990). This interest is likely that the analysis of teaching practices play a joint role between these two traditionally disjoint areas (Bali, 2004, 2005). At the same time, it is subject to different interpretations in terms of its implementation and the theoretical models that inspire, probably because of the diversity of issues that arouse his appeal. Analysis of teaching practices is used as a tool for both professional people (development related to step back from the action, knowledge production on the action skills), activities (identify and classify new practices to redefine the contours of a profession) and organizations (build the expertise of the organization and shared knowledge base system) (Hashweh, 1987). Indeed, in French literature, talking again "analysis practices" has not remained without contact with research on teacher thinking: "The analysis of professional practices is an integral part of the work of tomorrow because the reflective practice is a metacognitive position that has become a factor of change (Beillerot, 1998; Tochon, 2000, p 131). However, Tochon (2000): "Thinking covers, very broadly, any study on how teachers think, know, perceive, to represent their professions, disciplines, activities and, by extension, how they reflect on issues related to their daily teaching activities, how they solve their problems on their cognitive planning, their convictions, their personal history and their search for meaning" (Tochon, 2000 p 130). The Anglo-Saxon research undertaken in this field will change the idea that scientist's do what teachers need to know to optimize their teaching practices. These studies do not tell us what teachers need to know to teach, but shed light on the following question: what kind of knowledge they can teachers use to optimize their teaching practices?

\section{Object}

Because our questions research "implicit thoughts" as determinants of teaching practices of gymnastic teachers, we will define a first time teaching practices and check in a second time the relationship between teaching practices gymnastic teachers and their "implicit thoughts." 


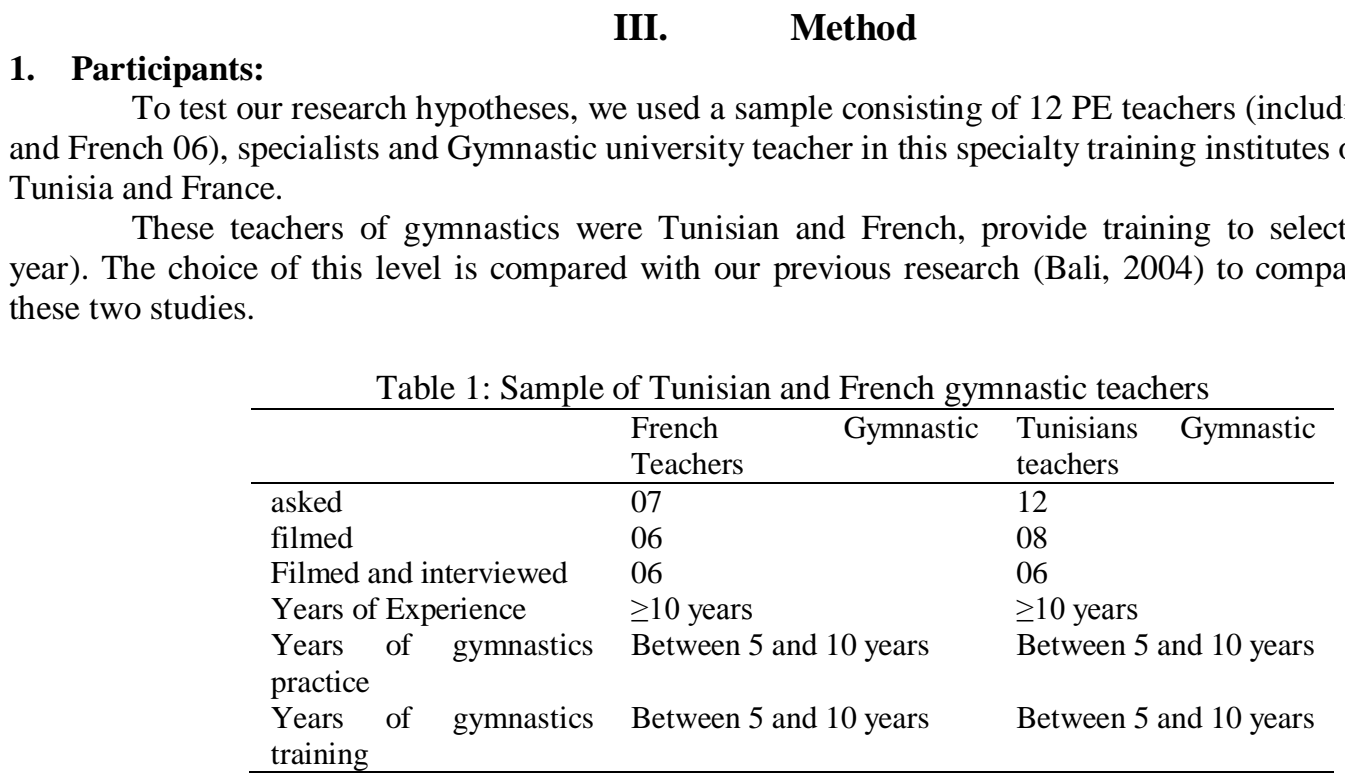

Three main reasons justified the choice of this sample: (i) an institutional reason: This research is part of the Franco-Tunisian cooperation and follows an agreement of co-supervision, which justifies the choice of Tunisian and French gymnastics teachers. This double origin, we will make a comparison between the teaching practices of teacher's Tunisian gymnastics and professional practices of teachers of French gymnastics, (ii) a cultural reason: There is a difference in culture between the two countries. This allowed us to hypothesize that this difference in culture implies a difference to the status of body and PE. This difference does not influence the risk educational practices of teachers of French and Tunisian gymnastics? And (iii) an academic reason: The initial training received by teachers of Tunisian and French gymnastics is different (Bali, 2004). According to the official program of initial training in Tunisia 50\% theory 50\% practice whereas France. $1 / 3$ theory $1 / 3$ pratique and third tools and method of pre-vocational and academic work.

To maximize opportunities to cover gymnastic teachers' intervention, we asked all teachers working in the Island of Paris France and Tunisia ISSEP are lecturers with first year classes (L1) to participate in this study. Our work sample meets these three parameters as recalled in the table below.

By means teaching practices, the set of behaviors undertaken by teachers in the classroom to teach, help students to learn, make decisions, adjust their courses and evaluate students. The Anglo-Saxon research undertaken in this field will change the idea that scientist's do what teachers need to know to optimize their teaching practices. These studies do not tell us what teachers need to know to teach, but shed light on the following question: what kind of knowledge they can teachers use to optimize their teaching practices?

\section{The interview audio post-session}

A week after the video recording sessions observing teaching practices of teachers of Tunisian and French gymnastics, a semi structured interview was conducted with each of the teachers observed and recorded. This interview was conducted with six teachers working in the Gymnastic university teacher ISSEP Tunisians and six teachers working in the French gymnastics UFR STAPS of France.

We then proceeded to the transcription of audio recordings of semi-structured interviews individually established after the observation of each teacher to form the body and we have reported in the grid also developed by the researcher (step 2). This grid has allowed us to categorize the implicit thoughts gym teacher asked about their own teaching practices. Finally, the third step is the linking of analyzes from different investigative techniques. We crossed the data collected during the first two stages. This categorization allowed us to approach through what the teacher said and done, the body design of its students. This comparison data is in aggregate form between the two (French and Tunisian) sample groups.

\section{The formation of the corpus}

Structured interviews: we used a dictaphone to record the responses of teachers interviewed. The questions focus on specific teaching practices previously filmed subjects interviewed in the first place. Then the gym teachers are interviewed while allowing them freedom of speech they can express themselves at their ease. Accordance with the principles of the semi structured interview (Mucchielli, 1979, 1983), the interview guide may change due to data collected by the observations of teaching practices in these subjects interviewed but 
without deviating from the main thesis slightly. The term was scheduled for 40 minutes, according to the same considerations, the duration varied slightly from one subject to another.

\section{Summary of the device talks}

Data collection was conducted in three phases. The first is an interview with the teacher in order to have data that can be useful in this study as the number of years of experience, his career as a gymnast and / or gymnastics coach. At this meeting we presented to the teacher, the various stages of this research namely a video recording of a regular practice session which dealt learning a semi gymnastic element is the round off followed by an interview directive in connection with the filmed meeting representing the other two phases of data collection.

\section{Result}

The results showed that the survey collected empirical (observation of teachers in classroom) that their practices are not always based on scientific considerations and biomechanical knowledge for example is rarely used in gym class. However, the observed teachers seem to prefer their metaphors and the style language of common sense. Indeed these teachers justify their teaching practices by the fact that the level of the students did not allow them to integrate science into the practice gym sessions.

Our results showed that the skills of teachers based in part on their perception of organized gymnastic movements. The majority of teachers seem further focused on motor learning problems, leaving thus, the problems of academic content that inform the learning, which seems to interest first and foremost, is the technical aspect or purely motor, these learning.

They thus, rely on metaphors in their technical explanations to make them more intelligible. However, when they are asked to justify their teaching practices, teachers rely on conceptual knowledge, essentially allusive, which means the absence of scientific referents compelling nature. In this fact the means refers the reader rather than the professional culture of teaching gymnastics consists of a set of meaning and understanding. interviews:

Comparison of the results of observations of teaching practices and Tunisian gymnastic teachers'

Therefore is possible to think that knowledge, at times, is not abandoned but merely suspended. The challenge is learning as we had already advanced, depending on the constraints of nature style of communication. The need to create conditions so that the student is or enrol in educational relationships and assumes the responsibility to teach himself led to temporary suspensions of knowledge, without however the intention teaching the teacher seems questionable.

\section{Discussion}

In our study we have indeed previously shown that the demonstration gesture (53.46\%) occupies an important place in the teaching practices of Tunisian gymnastic teachers observed. But they do not declare their educational interventions based on gestural demonstration that in a proportion of $20.44 \%$.

Also, in practice, Tunisian teachers gymnastics observed base their teachings on remediation and correction of students (42.21\%). But in the interview, these teachers say they use remediation and correction in a proportion of $11.33 \%$ of all statements of these teachers. Similarly, they declare their educational interventions based on technical knowledge (18.22\%), while the results of observations of teaching practices of these teachers have shown that they based their teachings on "explanation of gymnastic movements by technique "in an amount of $33.66 \%$.

These discrepancies may be explained in two ways:

- However, the teacher in question are not brooding enough to realize what actually they do on the ground and internalize it as a personal teaching practice as defined by Perrenoud (1994) in his work on the teaching profession "A real professionalization of the teaching profession means to ask practical questions but do so conceptually coherent" Huberman, 1985). Also, Perrenoud (1994) in his book: "The training of teachers between theory and practice," said that: "On the one hand, the job is done routines that the teacher operate relatively conscious, but without measuring their arbitrary, so without choosing and really master. Other moments of practice are an expression of habit's (cf. Bourdieu), a system of schemes of perception and action that is not under conscious control. Either because of the exigency, either shameful or unthinkable for practicality, the master does things he does not know or prefer not to see. Only realistic analysis of gestures in the art can permanently direct and guide their new training approach. Perrenoud talking about professional intervention practices and professionalization of teachers must bring the teacher to make a reflective analysis of practice and habit's in order to evolve. Change according to the same author goes through a transformation of the habits of the teacher as well as the provision of educational revenues. Determining the content of education 
implies a didactic analysis, ownership by students involves differentiated instructional interventions, and evaluation requires an appropriate methodology.

- Whether they are aware, but they eliminate a behaviour they are far too pragmatic and therefore scientific basis they are supposed to use.

By cons, it can be said that teachers base their lessons gymnastics on the so-called "trial and error" method, i.e. they allow students to act and intervene only when there is a problem. For them, the teaching process is limited to preparation and development of hardware and "normally students can learn!" as said one French teacher (FR2) and "If they fail then there teachers involved". We can say that it looks like a "trial - error" system.

The problem in this case appears in the role of the teacher in an EPS session that was the subject of several studies (Marsenach 1989; Perrenoud 1994). A debate within the profession about the role attributed to the teacher: Is it a design engineer with his own tools or can it only be a practitioner and user of tools developed by other adapter? For example, a doctor is a doctor who uses drugs designed and developed by laboratories. Its role is to make an accurate diagnosis, to establish formal relationships between the relevant remedies symptoms and causes of dysfunctions in order to obtain the healing of the patient. In any case, it designs the products they use. In other words, the teacher there sufficient training time and the conditions necessary to develop and validate situations that efficiency? Should the "didactics" (working or not with "practitioners") play this role? According to the theoretical choice is made, the proposed strategies initial and ongoing training will be different.

However, some authors (Marsenach 1991; Perrenoud, 1994), the action of the PE teacher is more like that of the "handyman" in the best sense of the term, it transforms and adapts continuously available tools in their immediate environment, but not provided for the task it has set itself. The all origin of these tools is various: research products unsuitable for teaching conditions, experts to the federal environment productions, situations experienced by colleagues in different contexts. Comparison of the results of observations of teaching practices and interviews of teachers of French gymnastics and discussion the analysis of the results of observations of teaching practices of teachers of French gymnastics and results of interviews with the teachers showed us a certain gap between these two elements we cite as an example:

The results of the all didactic observations teaching practices of French gymnastic teachers showed that they support their educational interventions by the "reinforcement learning" in a proportion of $12.78 \%$, while they report to base their teachings this same category as in an amount of $5.07 \%$.

Similarly, the "demonstration gesture" has a proportion of $18.82 \%$ in the teaching practices of gym teachers. But in interviews, these teachers say use gestures demonstrate that in a proportion of $10.98 \%$. While the "remediation" accounts for the lion's share in the educational practices of teachers of gymnastics $(59.21 \%)$, only $5.63 \%$ of the teachers surveyed said they base their teachings on remediation and correction of students.

This discrepancy could be explained in the same way as Tunisian gymnastic teachers (see previous paragraph).

The use of scientific vocabulary is not claimed and he notes a false necessity since it is sufficient to use the living words that exist in abundance in everyday language, words that have been proven, cover all current needs and have the advantage of being known? (Parlebas, 1981).

How to explain this state of affairs?

Therefore this is due, in our opinion, for two complementary reasons:

The nearly, universal between training and the athletic training of future teachers of the recent reform that separates these two formations confusion. Moreover, previous studies have shown that the separation of the two training physical education and sport (PES) in physical education (EP) and sport (S) since 1996) creates a real dilemma (Bali, 2004). The opinions are somewhat divided on the effectiveness of this split effect: teachers providing the theoretical course think that separation is pretty useless, while those responsible for the practical courses seem to approve. The reason given shed some light on the diversity of opinions about the usefulness of this split-level training. Some even claim a difference in the intellectual level an advantage to the first, confirmed academicians, including the classic academic regimen can be experienced by the "sport."

In our study shows some difficulty in statements of teacher's survey in structuring programs in a separate training course: a minority seems to lean towards a similar theoretical training content. Although, this population may still constitute a majority in the future of education as it is for teachers with the less than of ten of seniority has the highest hesitation. The (59\%) of teachers survey is training, in parallel, the other dies oriented students and report using "slightly different content" and "completely different content." (Ben Chaabane, 2007).

Despite the split dies and lack of program review, the faculty of Higher Institutes of Physical Education and Sport continue to ask the question: we do train sports coaches or future physical education teachers? Logically and even in fact, ISSEP form physical education teachers in the sector which has been the subject of our investigations. However, the training of future teachers of PE requires the teacher to teach them not simple motor behaviour (be they advanced) but teachable objects to be teachable, a driving behaviour must be 
understood, that is explained its physiological basis, biomechanical, anatomical, or a point of view of cognitive psychology ... It is this theoretical insight which also ensures better error correction. Finally, the theoretical insight characterizes the expert with a single running. These educational and epistemological considerations seemed almost absent teaching practices of teachers interviewed and observed for some.

This educational problem arises more widely the articulation between theory and practice. From the nineteenth century, science has been elevated to the status of science in favour of contributory social progress.

Hence the invention of the term "applied science" an applied science is a set of practical knowledge from theoretical knowledge which aims to improve social practices, as is the case of Medical Sciences, agricultural sciences, engineering, PE...

The integration of contributory sciences; (biomechanics, physiology, psychology ...) in the programs of Higher Institutes of Physical Education in France, confirmed this logic. Not having the same trend, our culture seems remained the dichotomy between theory and practice (Bali, 2005, 2013).

For this reason why the teaching practices observed among teachers working in the Tunisian ISSEP that seem to deny any link made between basic and applied science and motor learning, even if they say otherwise in the interviews for this research and another previous research (Bali, 2004 / Ben Chaabane, 2007).

Indeed, any observer can actually fail to notice the richness of sports vocabulary. According Parlebas Pierre (1981), the language of sport is like a treasure trove that every specialty feeds its own treasures. This vocabulary refers to techniques or tactics immediate action, it refers to what is carried himself as a practitioner, that it is run as a teacher or coach, or that we observe as a spectator: execution language, prescription or description. However, the practice of science is different and it is not to describe an activity but to build concepts. However, the motor execution can happen in this language, indispensable tool for scientific study (Parlebas PXII 1981).

However, the PE reflects a significant space in this area. The technical terms of the current language of sports illustrate a motley appearance, they respond to the use of "on the job". Created in practice and practice, they are indispensable, they often bear witness to the remarkable insights of man and land as is necessary, because they "stick" to reality. But they did not respond to questions of the research, as the language of the search cannot be that of the track and the jumper. The scientific lexicon is not opposed to the technical vocabulary and do not deny it, both are complementary.

1 - "The terminology concerns are they are quite far from the" realities "of the land and they do not show willingness to free and sterile abstraction? These concerns do they reflect trends "intellectualist" who turns their backs to practice? "

Tackle scholars in terms of the facts of mundane description may justly incur the reproach free abstraction, Parlebas (1981). In the same spirit, Peter Greco wrote an article about gymnastics on the crossing of sheep: "I do not think the use of assimilation and accommodation, which have such a wide use in educational, lights at all the analysis you will make learning a complex behavior. According to Parlebas (1981): "Retell mundane truths using sophisticated concepts do indeed bring no further explanation". Use reputable concrete terms such as "stride", "swing", "dive", "headline" is evolving according Parlebas in abstraction. It is true that some words refer to the field very "talking" to sensitive data, but it remains that any term of the technique is the result of an abstraction. Parlebas confirmed in the sense that the description and / or the analysis of some elements (pole vault in track and field, an output high bar in gymnastics) is to refer to a formal model which shows a concept.

2 - "Who does it serve to develop scholarly theories then it would suffice to simply name the obvious facts, are imposing themselves to the intuition of any observer, and that common sense can to recognize any natural way?

3 - The value of evidence of "experience" is particularly questionable. The latter occurs more as an obstacle as a guide. Indeed, Gaston Bachelard said: "The first experience can in no case be a safe support" (Bachelard, 1938, p 23). Everything we used to call "common sense", "experience", "intuition", "practical knowledge" in fact refers to the subjectivity of the individual, his passions. This subjective input and effervescent has indisputable fertility but that is not to be taken literally. However, according to Bachelard (1986): The assertion of the first intuition is an obstacle to scientific thought" (Bachelard, $1986 \mathrm{p} \mathrm{78).} \mathrm{Parlebas} \mathrm{deserves} \mathrm{to} \mathrm{be} \mathrm{recorded} \mathrm{in} \mathrm{"golden}$ letters" in front of physical education and sports facilities. However no energy this language which purports to be scientific is not it a common jargon? Is not this a pedantic language incomprehensible to the inexperienced, which seeks to scientist and complicated totally artificial way? "

The misuse of esoteric terms, the confusion between the language of the description and explanation of the excess of metaphorical language and generalities do not always save the work of physical education and sport (Parlebas, 1981). Indeed, according to this author, the language of the land is full of techniques, foreign and mysterious words, misunderstood by laymen. However, as any practice raises extended its specialized language: it is necessary to identify the name objects or usually actions ignored. This phenomenon is found in all human practices secreted their own action and communication technologies such as carpenters and judokas, notaries and judokas. The question arises: how the legendary simplicity of common sense it does accommodate 
miraculously and sometimes even on the ground, the use of terms such as "hamstring" and "stern-mastoid" or "circumduction"? Indeed, the terminology practitioners researchers in physical education and sport is obviously subject to the constraints of any language specialized and scientific purposes. Thus, as the surgeon invents a rich assortment for its multiple operations, the coach creates words and essential for transmission to his technical experience expressions, the researcher must pay practices drive terminology accuracy requirements and rigor imposed by the logic of his study. Thus, the charge of jargon addressed to him as opposed to "real" language of the land, does not seem to be legitimate according Parlebas (1981).

4 - "In the event that a scholarly terminology is necessary, it does not suffice then to borrow the basic disciplines to (biological sciences, humanities ...) concepts with a recognized scientific status long and the applied to sport and physical activity?"

The orbiting of physical education by the biological sciences has always seemed self-evident. The primacy of biomechanics is not the result of a need but the consequence of a contingent of body functioning. The magnitude of the historical process which imposed a body design machine was "apparent" and "natural" considered the anatomy and physiology as "basic science" (known in our science research contributory) they enough to apply to sport and physical activity. According to Parlebas (1981), while reflecting the specific requirements of its practice practitioner, the dominance given to the concepts and biological data is bound to raise eyebrows. Indeed, the biological imperialism sometimes triggered a violent backlash against the part of some physical education teachers. Thinking explain each sport specialty juxtaposing the views of the physiologist, psychologist and sociologist, we got a tangle of views with no major links between them (Parlebas, 1981: XXI). Deny the specific language of physical education is to deny the legitimacy to his field and refuse to speak to the teacher of physical education.

However, in earlier research Jacques Ulmann (1980) stated that physical education is primarily educational and should simply assign men goals. There can be no truth in PE because it is not a science and cannot be defined in terms of error and truth. He argues that PE cannot be a science because it has no welldefined area and continues legal purposes. For him if PE is a technique, it would be preceded by a theory, but theory and practice interfere.

Lamour (1989) address the issue of how the "theory and practice" in PE, which has, according to him in two faces: the act of teaching and the motor act, each one acting according to its specificity. In PE, teaching practice is inconceivable, according to the author, apart from the driving practice. they illustrates his point of view with a concrete example from the teaching practice: a teacher prepares a gym session saw the emergence of two levels of practice simultaneously: the level of the teaching act itself, since the teacher will set implement a set of exercises that target the ownership of a gymnastic element by the student and the level of the motor act that persists in the educational process in which the teacher is in the obligation to use his experience, technical accurate gymnastics this item. The author also describes the obstacles to the means of access "from theory to practice." These barriers are due firstly to training problems (being foremost practitioners, PE teachers are not naturally turned to the theory), secondly the fact that PE is been the double fragmentation: it undergoes one of the contributory sciences and that of his own techniques. Today, since the 90s, a comparative study was conducted in 2004 on training programs showed that the share of practices shall not exceed 1/3 of the training programs in the Faculty of STAPS while remaining $2 / 3$ are divided equally between theory and familiarity with the place of work (Bali, 2004). In the same research, she showed in a comparative study that was conducted on training programs, practice occupies $50 \%$ of the time devoted to the initial training of student teachers (Bali, 2004).

Since already 80, Parlebas (1985) proved of a triple destruction: practices, theories, and reference and areas of intervention. Thus, it supports the legitimacy of the science of motor action provided to abandon "the EPS to smithereens." Any pedagogy immersed in a system of values based on a design at least acknowledged human and social relations. According to this author, knowledge of sports techniques, motor learning, motor situations, and the practitioner is essential but that does not make it a science. Any physical activity or sport is a motor system interaction with their root lines drive essential units. Structural design of the motor action can apprehend (the simplex). The motor action is independent of the psychological or social biological determinants.

It is expressed by the internal logic (Parlebas 1985). However, Glazer (1994) distinguishes professions "high knowledge" occupations he calls "stars" and those "less prestigious" he considers incapable of developing a knowledge-based professional scientific and systematic. According to this author, the professions "stars" are based on a systemic basic knowledge that scientific knowledge is the model " Lechevestrier (1999), joined this analysis: "Clarifying the proper object of education, tossed decades between doctrinal rivalries, and give like Georges Demenÿ a scientific basis, that is the intention fed by Jean Le Boulch from the beginning of his career (Lechevestrier 1994). Moreover, the introduction of basic sciences in the training of practitioners (engineers, doctors, PE teachers ...) attests.

Dissimilar of these obstacles, this author believes are relatively surmountable, the irreducibility of the practice is an insurmountable obstacle scientific point of view, and it is on this point that it will focus its 
subsequent reflections. Thus, he indicates the ambiguity of the phrase "theorizing practice" was not the exact opposite of "practice theory". Donald Schön (1998) and Bali (2005) join the author asking the general question of the applicability of the theoretical sciences and professional practice shows that this joint has been made possible in the modern West with the positivist. Advocating the idea of social progress through science, Auguste Comte was able to demonstrate the ability to invest scientific discoveries in social activity, to promote the quality of life of citizens. The civil works undertaken at the end of the $19^{\text {th }}$ century throughout Europe, thanks to the expertise of scientists and engineers (we think the works of Gustave Eiffel in France, for example) can be seen. Other researchers are interested in the relationship between theory and practice in the professional life of teachers in general to the example Chartier (1998), which has two opposing models of these relationships: knowledge forged by researchers and fill rarely expectations practitioners who prefer information directly usable on the one hand and the "how" rather than the "why" other. The second model clearly separates the world of the theory of the practice: if the practice cannot use these theories are external, it must produce its own theory. Indeed, the author ends by emphasizing the common assumption in both models. "Concerning reports theory practice and professional learning, there view is: There is no theory without practice and without reflection on practice and this is true both in the workplace and in education" . And again: "a good theory, it is convenient ...". Indeed, the introduction of basic sciences in the training of practitioners (engineers, doctors, PE teachers ...) follows this logic.

\section{Conclusion}

Nerveless, we ask later if our culture still steeped in the dichotomy "between theory and practice" (Bali, 2005), can promote joint that positivism has promoted in the West in the late nineteenth century. For the positivism, as stated by Georges Demeney has some unfortunate consequences. In fact, it makes the PE teachers' anthropo-techniciens engineers "and endorsed the comments of his friend Pagés, who writes that" when man ... to accept it as a perfectible "animal", then it may benefit from the discoveries that have improved animal species are applying. "Moreover, Lahire (1998) involves a whole theory of action, reflexivity, knowledge and practice that is played by answering the question "how is it possible to ignore what are doing and what we know?".

Therefore, the problem, according to the author, does not lie in the fact that we do not know what we know and do, but rather in the fact that we do not always have good theoretical frameworks to talk about what we know and we do. Indeed, this explains the distortion that exists, according to the sociologist, between what actors do and actually know what they say and do and know. Few institutions "deliver" knowledge of work habits and are accompanied by a work of "transmission" of discursive frameworks, ideological ... etc...

In fact, one notices that everything happens as if the teaching and official discourse ideology lives an independent life parallel, with little relevance to educational practice. And this is where lies the role of the sociologist that will allow these players to take control of what they do and what they know, blending the negative effects of "bad framework" (Bali, 2013).

In light, this literature has amply demonstrated the importance of clarifying the determinants of teaching practices in physical education teachers in general and gymnastics in particular event and attitude instead of the past in their teaching practices. It is obviously in this role, fairly addressed by the publications that rank this research. We will try in this research to apply the findings from the epistemological studies on the determinants of educational practices among teachers of Tunisian and French gymnastics specific case of PE teacher training.

«A lucid teacher will deny the irreplaceable experience and intuition, two dimensions that previous scientific knowledge ». (Parlebas, $1981 \mathrm{P}: \mathrm{XVII})$.

\section{References}

[1]. Altet, M. (2003). Characterize, explain and understand teaching practices to contribute to their evaluation. Records of Educational Sciences, 10, 31-43.

[2]. Amade-Escot, Ch (2004). Study the work of the physical education teacher in the classroom: teaching contribution to the research analysis of actual practices. In J-F. Marcel (ed.), Practice teachers outside the classroom (pp. 53-77). Paris: L'Harmattan.

[3]. Astolfi, J-P (1998). The error, a tool for teaching. Paris: ESS.

[4]. Bali, N, (2004). Epistemological and didactic relationship between biomechanical knowledge and teaching gymnastics designs trainers and student teachers and teachers' practices, for ISSEP. Unpublished MSc thesis dissertation, University of Tunis.

[5]. Bali, N, (2005). "Theory and practice" Articulation in the training of Tunisian student physical education teachers. In the journal Research and Training. N49. (P.135-150).

[6]. Bali, N (2013). Teachers' thought processes: the case of Tunisian Gymnastic University Teachers. in Creative Education volume 4, Number 7, July.

[7]. Bali, N \& all. (2013). Heterogeneity language conceptions' physical education teachers of the fourth (4th) year of primary French schools in Tunisia. In IOSR Journal of Research \& Method in Education (IOSR-JRME). e-ISSN: 2320-7388,p-ISSN: 2320-737X Volume 1, Issue 4 (May. - Jun. 2013), PP 77-84

[8]. Charlier, \& Donnay (1994). Teaching at the University: a trade that can be learned. Edition: Brussels: DeBoeck.Cizeron, M. (2002). Beliefs PE teachers as cognitive anthropological basis of their expertise in teaching gymnastics. PhD thesis, University of Rennes.

[9]. Cizeron, M, Gal-Petitfaux, N (2003): Knowledge of action and justification of knowledge in teaching situation: the case of gymnastics. Published in the French Review of Education No. 143. NPRI: Publication Services. 
[10]. Ketele, J-M. (1991). What training for university teachers? Education. A collective work. General state of education. Louvain-laNeuve: BUILDS, p. 115-124.

[11]. Gauthier et al (1997). For a theory of pedagogy. Contemporary research on the knowledge of teachers. Sainte-Foy (Quebec): Presses de l'Université Laval.

[12]. Lahire, B (1998): Logical practices: the "doing" and "say on doing." Research and Training in Review No. 27.

[13]. Lechevestrier, H (1999). The work of Jean Le Boulch: The concern for consistency between science and teaching practice. In the EPS Review No. 279.Mucchielli, R (1979). The content analysis. Paris: ESF

[14]. Marsenach, J, (In 1989). "The practices of PE teachers in colleges". French Review of Education, n.89, p. 7-10.

[15]. Mucchielli, R (1983). The face-to-face. Paris: ESF.

[16]. Parlebas, P (1981). Contribution to a lexicon commented science of motor action. Paris: INSEP.

[17]. Parlebas, P. (1991). Didactic and internal logic of APS. EPS Journal, 228, 9-14.

[18]. Parlebas, P, Dugas, E (1998). Transfer of learning and field of motor action. In EPS Review No. 270, Paris, pp: 41 -49.

[19]. Piard, Cl (1982). Fundamentals of gymnastics. Paris: VIGOT.

[20]. Piard, Cl (1988). Instructional treatment and activity theory. In the Journal STAPS No. IX.Perrenoud, Ph (1994). Professionalization of the teacher profession, dual training and reflective practice. EPS in the Journal No. 250.

[21]. Perrenoud, $\mathrm{Ph}$ (2001). Développer la pratique réflexive dans le métier d'enseignant. Paris : ESF.

[22]. Schön, D-A (1994). The reflective Practitioner. Montreal: Editions Logics.

[23]. Shulman, L. (1986). Paradigms and research programs in the study of teaching. In M.C. Wittrock (ed.), Handbook of research on teaching (pp.3-36). New York: MacMillan.

[24]. Tochon, F (2000). Research on teacher thinking: a paradigm maturity. In French Review of Education No. 133. 